Tropical Journal of Pharmaceutical Research April 2015; 14 (4): 559-565

ISSN: $1596-5996$ (print); 1596-9827 (electronic)

(C) Pharmacotherapy Group, Faculty of Pharmacy, University of Benin, Benin City, 300001 Nigeria.

All rights reserved.

Available online at http://www.tjpr.org

Original Research Article

http://dx.doi.org/10.4314/tjpr.v14i4.1

\title{
Avanafil Liposomes as Transdermal Drug Delivery for Erectile Dysfunction Treatment: Preparation, Characterization, and In vitro, Ex vivo and In vivo Studies
}

\author{
Khaled Mohamed Hosny ${ }^{1,2 *}$ and Hibah Mubarak Aldawsari ${ }^{1}$ \\ ${ }_{1}^{1}$ Department of Pharmaceutics and Industrial Pharmacy, Faculty of pharmacy, King Abdulaziz University, Jeddah, Saudi Arabia, \\ ${ }^{2}$ Department of Pharmaceutics and Industrial Pharmacy, Faculty of Pharmacy, Beni Suef University, Beni Suef, Egypt \\ *For correspondence: Email: Elswaify2000@yahoo.com; Tel: +966592722634; Fax: +96626951696
}

Received: 18 December 2014

Revised accepted: 19 March 2015

\begin{abstract}
Purpose: To formulate avanafil, a recently approved phosphodiesterase-5 enzyme inhibitor, in liposomal form for enhanced transdermal permeation and bioavailability

Methods: Two preparation procedures were employed, leading to the formation of multilamellar vesicles (MLVs) and reverse-phase evaporation unilamellar vesicles (ULVs). The effects of the preparation method and lipid content on the encapsulation efficiency and particle size were studied. Studies assessing the stability, in vitro release, ex vivo permeation and in vivo bioavailability were also conducted in rats.

Results: The preparation of avanafil liposomes as MLVs, the addition of cholesterol, and the use of more rigid phospholipids all increased the avanafil encapsulation efficiency within the liposomes (95.61 $\%)$. The stability studies revealed that the liposomes prepared using phospholipids with higher transition temperatures (dipalmitoyl-L- $\alpha$-phospatidylcholine) were significantly more stable for a longer period of time after storage at $25 \pm 0.5{ }^{\circ} \mathrm{C}$ and $60 \pm 5 \%$ relative humidity for a period of 2 months $(p<0.05)$. In vivo pharmacokinetic results from rats showed a significant increase in the bioavailability of avanafil from transdermal liposomal formulations of up to 7 -fold $(p<0.05)$ compared to the topical drug suspension.

Conclusion: The developed avanafil liposomes represent a promising transdermal drug delivery system for the treatment of erectile dysfunction.
\end{abstract}

Keywords: Avanafil, Liposomes, Entrapment efficiency, Dipalmitoyl-L-a-phospatidylcholine, Erectile dysfunction

Tropical Journal of Pharmaceutical Research is indexed by Science Citation Index (SciSearch), Scopus, International Pharmaceutical Abstract, Chemical Abstracts, Embase, Index Copernicus, EBSCO, African Index Medicus, JournalSeek, Journal Citation Reports/Science Edition, Directory of Open Access Journals (DOAJ), African Journal Online, Bioline International, Open-J-Gate and Pharmacy Abstracts

\section{INTRODUCTION}

Avanafil was recently approved by two international pharmaceutical regulating bodies, the United States Food and Drug Administration (USFDA) and the European Medicines Agency (EMA) [1]. Avanafil is a phosphodiesterase-5 enzyme inhibitor and a second-generation erectile dysfunction medication [2,3]. Avanafil has advantages over other PDE5 inhibitors, as it is more selective for the PDE5 isoenzyme and a variety of cellular targets than sildenafil and tadalafil [4].

Avanafil is slightly soluble in ethanol, practically insoluble in water, and soluble in $0.1 \mathrm{~mol} / \mathrm{L}$ hydrochloric acid, and it undergoes a first pass effect. Therefore, the enhancement of its bioavailability is essential for the development of effective clinical formulations [4]. 
The skin, due to its structure, is considered a complex organ for drug delivery. Although the stratum corneum is considered the most important barrier to drug absorption through the skin, in recent years, transdermal drug delivery has been used to overcome the problems associated with skin barrier properties $[5,6]$. The utilization of novel delivery systems, including vesicular drug delivery systems such as liposomes, has recently been proposed [7]. These novel systems exhibit superior pharmacological properties [8]. Their activity depends on various factors, including their rigidity, lipid membrane composition, and encapsulation efficiency [9]. The use of vesicular drug delivery systems has been studied for both oral and dermal applications [10].

Therefore, the aim of this research was to prepare avanafil in liposomes to enhance its transdermal permeability and bioavailability for the treatment of erectile dysfunction.

\section{EXPERIMENTAL}

\section{Materials}

Avanafil was purchased from Jinlan PharmDrugs Technology Co., Ltd. [Hangzhou, China]. The egg phosphatidylcholine (PC), Lecithin, and cholesterol $(\mathrm{CH})$ were purchased from Sigma Chemical Company (St Louis, USA). DipalmitoylL-a-phospatidylcholine (DPPC) was purchased from Fluka Chemical Company (Buchs, Switzerland). All other reagents were commercially available and of analytical grade.

\section{Preparation of multilamellar liposomal vesicles (MLVs)}

Avanafil MLVs were prepared using the lipid film hydration technique [11]. $50 \mathrm{mg}$ of avanafil, and $100 \mathrm{mg}$ of lipid composed of phospholipid, lecithin, and cholesterol in different ratios, were solubilized in $10 \mathrm{ml}$ mixture of chloroform and methanol (1:1 ratio) in a round-bottom flask. The organic solvent was evaporated at $33{ }^{\circ} \mathrm{C}$ on a rotary evaporator (Buchi R-110 Rotavapor, Switzerland). Liposomes were prepared by dispersing the lipid component film in an isotonic phosphate-buffered saline solution $\mathrm{pH} 7.4$ (10 $\mathrm{ml})$. This procedure was carried out at $55{ }^{\circ} \mathrm{C}$ under mechanical stirring. The liposomal suspension was left to mature overnight at $4{ }^{\circ} \mathrm{C}$ to ensure full lipid hydration. Finally, the liposomal suspension was passed 5 times through a $4.5 \mu \mathrm{m}$ microporous filter and 10 times through a $2.2 \mu \mathrm{m}$ microporous filter for purification and kept in a refrigerator.
Preparation of reverse-phase evaporation (REVs) liposomes

Avanafil unilamellar vesicles (ULVs) were prepared using the reverse-phase evaporation technique [12]. $100 \mathrm{mg}$ of the lipid component, composed of phospholipid, lecithin, and cholesterol in different ratios, were solubilized in a $10 \mathrm{ml}$ mixture of chloroform and methanol (1:1 $\mathrm{v} / \mathrm{v})$. A thin lipid film was formed and dissolved in $10 \mathrm{ml}$ ether and $10 \mathrm{ml}$ acetone solution containing $50 \mathrm{mg}$ avanafil and $10 \mathrm{ml}$ phosphate buffer saline (PBS, pH 7.4). Sonication was continued for approximately $30 \mathrm{~min}$ until the organic solvent was evaporated. The liposomes were allowed to equilibrate at room temperature, and $10 \mathrm{ml}$ PBS was added to the liposomal suspension and passed five times through a 4.5$\mu \mathrm{m}$ and 10 times through a 2.2- $\mu \mathrm{m}$ microporous filter for purification. Samples were stored in a refrigerator overnight.

\section{Determination of the avanafil encapsulation efficiency in liposomes}

The free drug component was removed from the liposomal suspension by centrifugation for 45 min. at $15000 \mathrm{rpm}$ (Beckman Coulter Inc, Fullerton, CA). The concentration of (AVA) in the supernatant was quantified by HPLC with an Agilent 1200 series equipped with a UV diode array detector. A reversed-phase column $(25 \mathrm{~cm}$ $\times 4.6 \mathrm{~mm}$ (i.d.) C18, $5 \mu \mathrm{m}$ - Intersil[ ODS-3 (GL Sciences Inc, Japan)) was used. The column effluent was monitored with a UV detector at 230 $\mathrm{nm}$, and the eluent flow was determined to be 1.3 $\mathrm{ml} / \mathrm{min}$. The mobile phase included acetonitrile and methanol in a $0.05 \mathrm{M}$ ammonium acetate buffer, pH $2.5(20: 20: 60 \mathrm{v} / \mathrm{v} / \mathrm{v})$. The AVA retention time was $5 \mathrm{~min}$. The encapsulation efficiency (EE, \%) was calculated as in Eq 1.

$E E(\%)=\left\{\left(1-A_{F}\right) / A_{T}\right\} 100$

where $A_{F}$ and $A_{T}$ represent the free and total avanafil content, respectively.

EE was used to evaluate the effect of the preparation method, lipid composition, and type of charge on the encapsulation of avanafil in liposomes.

\section{Size analysis of liposomes}

To test the droplet size and zeta potential of the avanafil liposomes, $100 \mu \mathrm{L}$ of each liposomal suspension was diluted in $20 \mathrm{~mL}$ of distilled water. The mean droplet size and zeta potential of the resulting dispersion was determined by 
dynamic light scattering using a Zetatrac machine from Microtrac Inc. (PA, USA).

\section{Studies on drug-leakage from vesicles}

The different liposomal formulations were sealed in $30-\mathrm{mL}$ vials and stored at various temperatures $\left(4-8{ }^{\circ} \mathrm{C}, 25 \pm 0.5{ }^{\circ} \mathrm{C}\right.$ and $60 \pm 5 \%$ relative humidity) for a period of two months. Samples from each formula at each temperature were withdrawn at defined time intervals. The stability was evaluated by comparing \% EE for each liposomal suspension.

\section{In vitro drug release studies}

In vitro release profiles of avanafil liposomals and aqueous dispersions were performed in an openended cylindrical tube with one end tied with a dialysis membrane. Each sample being tested was placed in an open-ended cylinder and suspended in $250 \mathrm{ml}$ of phosphate buffer receptor medium ( $\mathrm{pH} 7.4)$ in a beaker placed on a thermostat magnetic stirrer and constantly stirred at $50 \mathrm{rpm}$ and $37 \pm 1{ }^{\circ} \mathrm{C}$. Sample aliquots $(5 \mathrm{ml})$ were withdrawn from the receptor compartment at predetermined time intervals of $5,15,30,60,120,180$, and $240 \mathrm{~min}$. After the collection of each sample, the same volume of fresh medium was added. The avanafil content in the withdrawn samples was estimated by HPLC, as described earlier.

\section{Ex vivo skin permeation studies}

Full thickness skin samples $(3 \times 3 \mathrm{~cm})$ were excised from the abdominal region of shaved male Wistar rats. The samples were freed from any subcutaneous fat and examined using a magnifier to assure skin integrity. The prepared skin was mounted between the donor and receptor compartments of the diffusion cells, with the dermal side in direct contact with the receptor medium. The amounts of permeated avanafil from the F7 formula and the pure drug were determined using HPLC.

\section{Skin-retention studies}

After conducting the ex vivo permeation study, the skin pieces mounted on the diffusion cells were removed. The remaining formulation adhering to the skin samples was scraped off, and the skin pieces were wiped with tissue paper. Each cleaned skin piece was cut and shaken in methanolic solution for $24 \mathrm{~h}$ to ensure complete drug extraction. The filtrate was removed and analysed using HPLC.

\section{Bioavailability studies}

Bioavailability analysis was conducted in rats in an attempt to explore the capability of the transdermal liposomal formulations in improving avanafil's pharmacokinetic parameters. Male Sprague Dawley rats weighing $\sim 300 \mathrm{~g}$ were used for the experiments. The rats were fasted for 12 hours prior to administering the drug formulation. The rats were given free access to water.

All experimental protocols were conducted after being approved by the Animal Ethics Committee of King Abdulaziz University, Jeddah KSA, ensuring that the care and use of all animals conformed to the European Union (EU) Directive. The animals were divided into two groups $(n=$ 6): the avanafil liposomal suspension group (group I), and the avanafil aqueous suspension group (group II). After trimming the back hair in one square spot, the F7 formula and the aqueous avanafil suspension were applied transdermally at a dose equivalent to $10 \mathrm{mg} / \mathrm{kg}$. At predetermined time intervals, blood samples of $0.2 \mathrm{ml}$ were withdrawn using a heparinized tube by retro-orbital puncture. Blood samples were then centrifuged at $10,000 \mathrm{~g}$ for $8 \mathrm{~min}$ to separate the plasma. The samples were then stored in sterile polypropylene vials at $-20{ }^{\circ} \mathrm{C}$. The resulting plasma drug concentrations were analysed by HPLC.

\section{Statistical analysis}

Differences among the group means were analysed for statistical significance using oneway ANOVA followed by the Tukey-Kramer Multiple Comparisons Test. Differences were considered significant at $p<0.05$. Statistical analysis was performed using SPSS for Windows (SPSS Inc, Chicago, IL, USA).

\section{RESULTS}

\section{Encapsulation efficiency (EE)}

Table 1 shows that the EE had higher values for the MLVs than for the ULVs of the same composition and molar ratio. Furthermore, EE had higher values for the DPPC-containing liposomes than for the egg phosphatidylcholine (PC)-containing liposomes of same molar ratio. Concerning the effects of the cholesterol content on the EE\% of avanafil in liposomes, the results showed that the EE\% can be increased by increasing the cholesterol $(\mathrm{CH})$ content to a certain limit, above which the EE\% decreases when the $(\mathrm{CH})$ concentration increases. 
Table 1: Particle size and encapsulation efficiency of avanafil multilamellar and unilamellar liposomes

\begin{tabular}{|c|c|c|c|c|c|c|}
\hline $\begin{array}{l}\text { Batch } \\
\text { code }\end{array}$ & $\begin{array}{l}\text { Liposomal lipid } \\
\text { composition }\end{array}$ & $\begin{array}{l}\text { Molar } \\
\text { ratio }\end{array}$ & $\begin{array}{c}\text { Mean MLVs } \\
\text { encapsulation } \\
\text { efficiency }( \pm S D)\end{array}$ & $\begin{array}{c}\text { ULVs } \\
\text { encapsulation } \\
\text { efficiency ( } \pm S D)\end{array}$ & $\begin{array}{c}\text { MLVs } \\
\text { particle } \\
\text { size } \\
(\mu \mathrm{m})\end{array}$ & $\begin{array}{c}\text { ULVs } \\
\text { particle } \\
\text { size } \\
(\mu \mathrm{m})\end{array}$ \\
\hline F1 & PC:Lec:CH & $3: 1: 1$ & $43.51 \pm 1.61$ & $23.51 \pm 0.92$ & 0.603 & 0.325 \\
\hline $\mathrm{F} 2$ & PC:Lec:CH & $3: 1: 2$ & $59.73 \pm 1.88$ & $35.40 \pm 1.02$ & 0.681 & 0.396 \\
\hline F3 & PC:Lec:CH & $3: 1: 3$ & $72.15 \pm 1.56$ & $49.92 \pm 1.41$ & 0.722 & 0.437 \\
\hline $\mathrm{F} 4$ & PC:Lec:CH & $3: 1: 4$ & $61.26 \pm 1.81$ & $32.13 \pm 1.16$ & 0.698 & 0.488 \\
\hline F5 & DPPC:Lec:CH & $3: 1: 1$ & $53.17 \pm 1.92$ & $31.62 \pm 1.53$ & 0.662 & 0.372 \\
\hline F6 & DPPC:Lec:CH & $3: 1: 2$ & $78.22 \pm 2.10$ & $47.61 \pm 1.69$ & 0.791 & 0.411 \\
\hline F7 & DPPC:Lec:CH & $3: 1: 3$ & $95.61 \pm 2.31$ & $60.01 \pm 1.72$ & 0.864 & 0.565 \\
\hline F8 & DPPC:Lec:CH & $3: 1: 4$ & $86.42 \pm 1.98$ & $53.12 \pm 1.92$ & 0.813 & 0.503 \\
\hline
\end{tabular}

Note: $P C=$ phosphatidylcholine; $C H=$ cholesterol; $D P P C$, dipalmitoyl-L- $\alpha$-phospatidylcholine.; Lec = Lecithin; $M L V s=$ multilamellar vesicles; ULVs = unilamellar vesicles. Data represent mean $\pm S D(n=3)$

The above results clearly show that liposomes prepared as MLVs with a lipid composition of DPPC:Lecithin: $\mathrm{CH}$ in a molar ratio of $3: 1: 3$ had a higher EE \% $[95.61 \pm 2.31]$ than all other liposome formulations.

\section{Size profile of liposomes}

Table 1 shows the particle sizes of the liposomes. The mean particle diameter of the MLVs was larger than that of the ULVs.

\section{Drug-leakage}

The results indicate that the liposomal formulation prepared with egg phosphatidylcholine (PC) was withdrawn from the study after one month because storage at 25 ${ }^{\circ} \mathrm{C}$ led to a substantial loss (75 \%) of avanafil. In the case of the liposomal formulation prepared with dipalmitoyl-L-a-phospatidylcholine (DPPC), the drug leakage from vesicles was less than 5 $\%$ at the end of test period, indicating the stability of these formulations (Table 2).

\section{Ex Vivo Permeation}

Figure 1 shows the transdermal permeation profile of avanafil in the $1 \%$ drug suspension and formula F7. The drug permeation levels after 12 $\mathrm{h}$ for the liposomal suspension and pure drug suspension were significantly different $(p<0.05)$. The cumulative amount of avanafil permeated from liposomal dispersion after $12 \mathrm{~h}$ was $2615 \pm$ $127 \mu \mathrm{g}(52.30 \%)$, while the cumulative amount permeated from the avanafil suspension was only $613 \pm 41(12.36 \%)$.

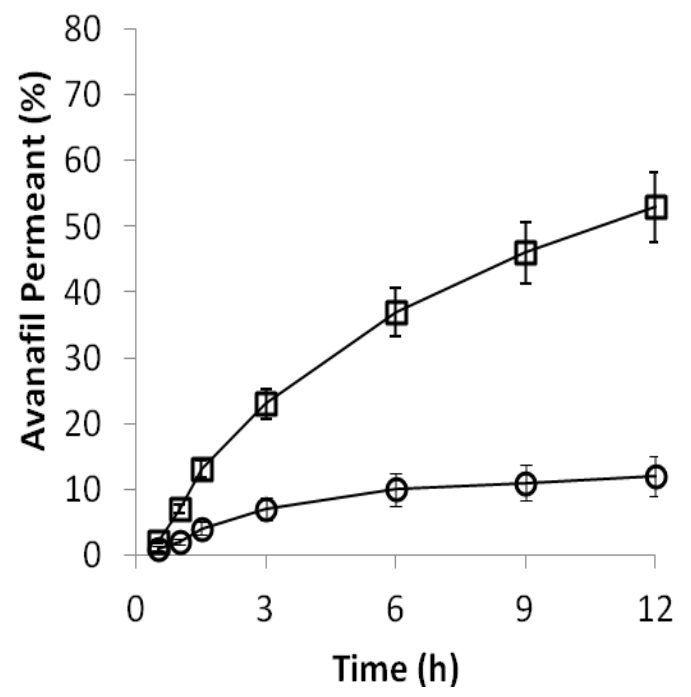

Figure 1: Cumulative rat skin permeation of avanafil from the F7 MLVs ( $\square$ ) and the aqueous avanafil suspension (०)

Table 2: Effect of storage at $25 \pm 0.5^{\circ} \mathrm{C}$ and $60 \pm 5 \% \mathrm{RH}$ (relative humidity) for a period of 2 months on the mean vesicle size and entrapment efficiency of batches F3 and F7

\begin{tabular}{ccccc}
\hline $\begin{array}{c}\text { Storage } \\
\text { period } \\
\text { (days) }\end{array}$ & $\begin{array}{c}\text { Particle size } \\
(\boldsymbol{\mu m})\end{array}$ & $\begin{array}{c}\text { Entrapment Efficiency } \\
(\%)\end{array}$ & $\begin{array}{c}\text { Particle size } \\
(\boldsymbol{\mu m})\end{array}$ & $\begin{array}{c}\text { Entrapment } \\
\text { efficiency }(\%)\end{array}$ \\
\hline 0 & 0.722 & $72.15 \pm 1.56$ & 0.864 & $95.61 \pm 2.31$ \\
15 & 1.212 & $44.27 \pm 1.31$ & 0.881 & $93.23 \pm 2.28$ \\
30 & 2.754 & $18.37 \pm 0.95$ & 0.912 & $92.11 \pm 2.05$ \\
45 & - & - & 0.931 & $91.50 \pm 1.97$ \\
60 & - & - & 0.952 & $90.97 \pm 1.89$ \\
\hline
\end{tabular}




\section{Skin-retention}

The results of the skin retention study indicate that the $\%$ of avanafil retained within the skin in case of formula F7 was higher than that from the aqueous avanafil suspension (25 and $4 \%$, respectively).

\section{Bioavailability}

The plasma concentration-time profiles for avanafil after the transdermal administration of liposomal formulation and aqueous suspension are shown in Figure 2. The pharmacokinetic parameters for all groups were calculated using Kinetica software (Kinetica 5.0.11, Thermo Fisher Scientific Inc, Waltham, MA USA) (Table 3 ). The AUC of the liposomal formulation was found to be $127.45 \mathrm{ng} / \mathrm{ml} . \mathrm{hr}$, which was significantly $(p<0.05)$ greater than that of the drug suspension $(17.23 \mathrm{ng} / \mathrm{ml} . \mathrm{hr})$. When the Cmax values were compared, a significant increase was observed in the liposomal formulation relative to the aqueous suspension ( $p$ $<0.05$ ).

\section{DISCUSSION}

The results revealed that the avanafil encapsulation efficiency in the different prepared liposomal formulations varied depending on the method of preparation and the lipid composition used in the prepared liposomes. The method of liposome preparation also had an effect on the encapsulation efficiency of avanafil. EE had higher values in MLVs than in ULVs of the same composition and molar ratio. This may be because the liposomes were prepared at a $\mathrm{pH}$ of 7.4, and avanafil has $\mathrm{pH}$-dependent solubility, with minimum solubility in basic medium. This, a higher $\mathrm{pH}$ should increase the unionized fraction of the drug, which is lipophilic in nature. Because the MLVs contained multiple lamellae capable of loading a higher mass of lipophilic drug than the ULVs [13], EE had higher values in the MLVs than in the ULVs of the same composition and molar ratio.

Concerning the effect of the type of phospholipids on EE, the results showed that the EE\% had higher values in the DPPC-containing liposomes than the egg PC-containing liposomes of the same molar ratio. This result may be because the encapsulation of avanafil depends on the fluidity of the phospholipid bilayer; the lower the fluidity, the greater the EE\%. DPPC is a synthetic product with a higher phase transition temperature $\left(42.5 \pm 0.25^{\circ} \mathrm{C}\right)$ than egg PC $\left(-7^{\circ} \mathrm{C}\right)$ [14], so the fluidity of DPPC is less than that of egg PC.

Table 3: Pharmacokinetic parameters of avanafil after the transdermal administration of $10 \mathrm{mg} / \mathrm{kg}$ of formula F7 and $10 \mathrm{mg} / \mathrm{kg}$ of avanafil aqueous suspension. The significance level was set at $p<0.05$

\begin{tabular}{|c|c|c|c|c|c|}
\hline Formulation & $T_{\max }(h)$ & $C_{\max }(\mathrm{ng} / \mathrm{ml})$ & $\begin{array}{c}\text { AUC }_{0-\mathrm{t}} \\
\text { (ng.h/ml) }\end{array}$ & $\begin{array}{c}\text { MRT } \\
(\mathrm{hr})\end{array}$ & $\mathrm{AUC}_{0-\infty}(\mathrm{ng} \cdot \mathrm{h} / \mathrm{ml})$ \\
\hline $\begin{array}{c}\text { F7 } \\
\text { Avanafil }\end{array}$ & $3.5 \pm 0.4$ & $8.42 \pm 0.89$ & $108.12 \pm 8.23$ & $9.23 \pm 1.01$ & $127.45 \pm 10.4$ \\
\hline $\begin{array}{l}\text { aqueous } \\
\text { suspension }\end{array}$ & $1.5 \pm 0.2$ & $3.64 \pm 0.21$ & $13.10 \pm 1.61$ & $2.15 \pm 0.21$ & $17.23 \pm 2.42$ \\
\hline
\end{tabular}

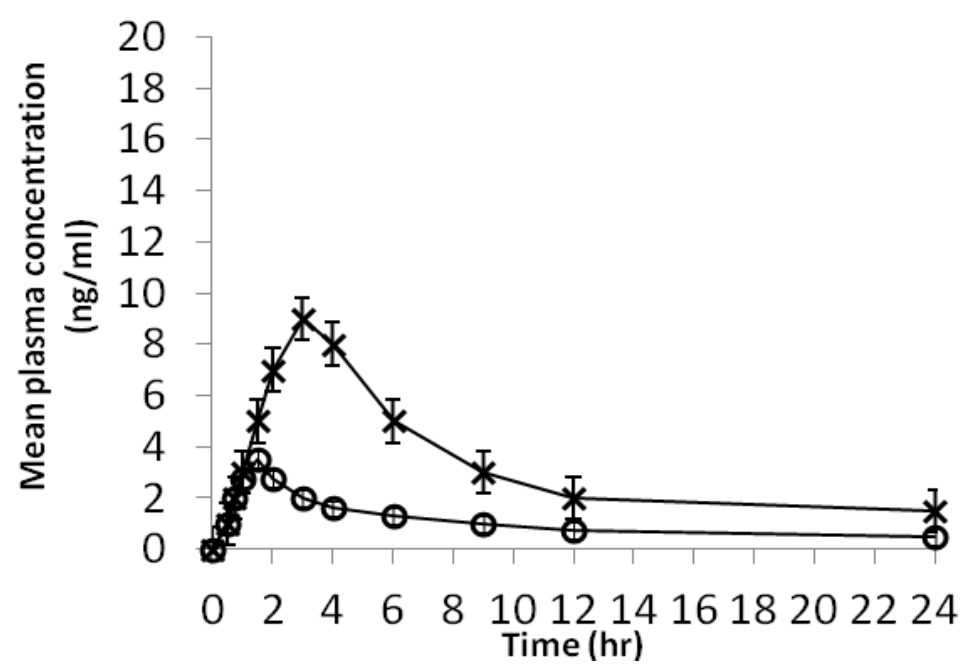

Figure 2: Plasma-concentration time curve of avanafil after the transdermal application of a $10 \mathrm{mg} / \mathrm{kg}$ dose of Batch $\mathrm{F} 7(*)$ or a $10 \mathrm{mg} / \mathrm{kg}$ dose of the aqueous avanafil suspension $(\circ) ; p<0.05$ 
This could lead to the increased EE \% in the liposomes containing DPPC. Concerning the effect of the cholesterol content on the EE \% of avanafil in liposomes, our results showed that the EE\% could be increased by increasing the $(\mathrm{CH})$ content to a certain limit. However, above that limit, the EE\% actually decreased with further increases in the $(\mathrm{CH})$ concentration. This is because cholesterol increases the rigidity of the bilayer. A more rigid bilayer results in a higher stability and reduces the permeability of the liposomal membrane [15], increasing the drug retention [16]. After a certain concentration, cholesterol can disrupt the regular linear structure of the liposomal membrane [17].

The mean particle diameter of the MLVs was larger than that of the ULVs. This was due to the presence of several phospholipid bilayers in MLV liposomes, leading to an increase in their mean particle size. These results can be attributed to the higher EE\% of the MLVs, which increases the spacing between adjacent layers, resulting in the formation of larger liposomes than in the ULVs [18].

The better stability of the DPPC liposomes compared with the PC liposomes may be attributed to the high phase-transition temperature of DPPC $\left[42.5^{\circ} \mathrm{C}\right]$ relative to the low phase-transition temperature of $\mathrm{PC}\left(-7{ }^{\circ} \mathrm{C}\right)$. Thus, the fluidity of DPPC is less than egg PC, leading to a decrease in drug leakage in liposomes prepared with DPPC.

The results of the ex vivo permeation studies indicated that the liposomal suspensions showed a 4 -fold higher permeation \% than aqueous suspension. This may be explained by the fact that liposomes can adsorb to the skin surface and transfer membrane-associated drug directly to the cell membrane, thereby facilitating drug passage across the skin [19]. Another possible reason is the lower solubility of avanafil at $\mathrm{pH}$ 7.4; at this $\mathrm{pH}$, avanafil is present in its unionized form, producing a cloudy solution [20]. In this form, avanafil is insoluble, leading to decreased permeation. In order for drug permeation to occur, the drug must first be present in the soluble form, either in aqueous or lipid solution. This leads to a decrease in the \% permeated from the aqueous avanafil suspension compared to the avanafil liposomal suspension, in which the drug remained solubilized in either the aqueous phase or the lipid bilayers of the liposomes.

The results of skin retention test could be attributed to the high retention of the drug in vesicular systems due to its intrinsic liposome- skin interaction behaviour. These results were in accordance with several research studies showing that liposome-associated drugs can be better routed into the skin [21-23]. The poor retention of the aqueous drug dispersion may be due to the presence of drug in an insoluble form, which acts as a barrier for its penetration and retention within skin layers.

The enhanced bioavailability of the liposomal formulations after transdermal administration is likely due to a combination of the following factors. First, liposomal vesicles provide a delivery vector by which avanafil can cross the skin barrier. Second, liposomal vesicles can introduce avanafil as a fine dispersion, unlike the coarse particles delivered in a standard drug suspension, with a correspondingly increased surface area and a reduced diffusion path length. Third, a higher adhesion surface contact between the liposomal vesicles and absorption site was afforded. When the MRT of the prepared liposomal formulation was compared with that of aqueous suspension, the liposomal was found to be higher than that of the aqueous suspension. This result could be ascribed to the retention of liposomes within the skin layers for a longer period of time, allowing them to serve as a drug reservoir for extended release into the viable epidermis over several hours. The relative bioavailability of the liposomal formulation was 739.6 with respect to the drug suspension. Thus, there was a 7.3-fold increase in the avanafil bioavailability of the drug in the liposomal formulation.

\section{CONCLUSION}

Suitable liposomal transdermal delivery systems of avanafil for potential use in the treatment of erectile dysfunction have been developed. The use of multilamellar vesicles and the incorporation of cholesterol and more rigid phospholipids both increase the encapsulation efficiency of the drug within the liposomes. The bioavailability of avanafil in its transdermal liposomal formulation was 7.3-fold compared to the topical drug suspension. The developed formulation is therefore a promising transdermal drug delivery system for the treatment of erectile dysfunction.

\section{REFERENCES}

1. Sanford M. Avanafil: a review of its use in patients with erectile dysfunction. Drugs Aging 2013; 30: 853-862.

2. FDA approves Stendra for erectile dysfunction 2014 
3. European Medicines Agency, Committee for Medicinal Products for Human Use CHMP assessment report. EMA 321885, 2013.

4. Fahmy U, Ahmed O, Hosny K. Development and evaluation of avanafil self-nanoemulsifying drug delivery system with rapid onset of action and enhanced bioavailability. AAPS PharmaSciTech 2015; 16(1): 53-58.

5. Ah C, Choi K, Choi K, Ki M, Bae H. A novel transdermal patch incorporating meloxicam: in vitro and in vivo characterization. Int J Pharm 2010; 385: 12-19.

6. Bariya H, Gohel C, Mehta A, Sharma P. Microneedles: an emerging transdermal drug delivery system. The Journal of pharmacy and pharmacology 2012; 64: 11 29.

7. Hosny K, Banjar Z. The formulation of a nasal nanoemulsion zaleplon in situ gel for the treatment of insomnia. Expert Opin Drug Deliv 2013; 10: 10331041.

8. Bangham $D$, Standish $M$, Watkins C. Diffusion of univalent ions across the lamellae of swollen phospholipids. J. Mol. Biol. 1965; 13: 238-252.

9. Gregoriadis G, Florence A. Liposomes in drug delivery: clinical, diagnostic and ophthalmic potential. Drugs 1993; 45: 15-28.

10. Farkas E, Schubert R, Zelk'o R. Effect of cholesterol on the characteristics of vesicular gels containing chlorhexidine. Int. J. Pharm. 2004; 278: 63-70

11. Furneri P, Fresta M, Puglisi G, Tempera G. OfloxacinLoaded Liposomes: In Vitro Activity and Drug Accumulation in Bacteria. Antimicrobial Agent and Chemotherapy 2000; 2458-2464.

12. Szoka F, Papahodjopoulos D. Procedure for preparation of liposomes with large internal aqueous space and high capture by reverse-phase evaporation. Proc. Natl. Acad. Sci. U.S.A. 1978; 75: 4194-4198.
13. Morilla J, Benavides $P$, Lopez O, Bakas L, Romero $L$. Development and in vitro characterization of a benznidazole liposomal formulation. Int $J$ Pharm. 2002; 249: 89-99

14. Perugini $P$, Pavanetto F. Liposomes containing boronophenylalanine for boron neutron capture therapy. J. Microencapsul. 1998; 15: 473-483

15. New C. Liposomes: A Practical Approach. Oxford University Press, Oxford. 1990.

16. Gulati $M$, Grover $M$, Singh $M$, Singh S. Study of azathioprine encapsulation into liposomes. J. Microencapsul. 1998; 15: 485-494

17. Rania M, Samar M, Nahed M, Ahmed G. Liposomes as an Ocular Delivery System for Acetazolamide: In Vitro and In Vivo Studies. AAPS Pharm. Sci. Tech. 2007; 8(1): 1-12

18. Gruner M. Materials properties of liposomal bilayers. In: Ostro, M.J. [Ed.], Liposomes From Biophysics to Therapeutics. Marcel Dekker, New York, 1987; 1-38

19. Choi H, Jung J, Ryu J, Yoon S, Oh Y, Kim C. Development of in-situ gelling and mucoadhesive acetaminophen liquid suppository. Int. J. Pharm. 1998; 165: 33-44

20. Agarwal R, Katare $P$, Vyas S. Preparation and In vitro Evaluation of Liposomal/Niosomal Delivery Systems for Antipsoriatic Drug Dithranol. Int. J. Pharm. 2001; 228: 43-52.

21. Fang J. Effect of Liposomes and Niosomes on Skin Permeation of Enoxacin. Int. J. Pharm. 2001; 219: 61-72.

22. Du Plessis J. The Influence of Particle Size of Liposomes on the Disposition of Drug into Skin. Int. J. Pharm. 1994; 103: 277-282.

23. Sentjurc M, Vrhovnik K, Kristl J. Liposomes as a Topical Delivery System: The Role of Size on Transport Studied by the EPR Imaging. Method. J. Controlled Release 1999; 59: 87-97. 